\title{
Magnetic anisotropy of asbestos fibers
}

\author{
Z. Ulanowski ${ }^{\mathrm{a})}$ and P. H. Kaye \\ Science and Technology Research Centre, University of Hertfordshire, Hatfield, \\ Hertfordshire AL10 9AB, United Kingdom
}

(Received 31 July 1998; accepted for publication 23 December 1998)

\begin{abstract}
The anisotropy of magnetic susceptibility of single asbestos fibers is measured. The alignment of both chrysotile and crocidolite fibers in magnetic fields is found to be due to the anisotropy. The average measured anisotropy of volume susceptibility is $0.40 \times 10^{-6}$ for chrysotile and $83 \times 10^{-6}$ for crocidolite. Fiber shape effects are estimated to contribute, on average, about $10 \%$ and $6 \%$, respectively, to the total anisotropy of the two types of fiber. There is no evidence of significant permanent magnetic moments. The magnitude of the observed alignment makes the effect potentially useful in real-time detection of airborne asbestos fibers. The experimental technique developed in the study can be used for measuring the anisotropy of small particles of well-defined shape. High sensitivity of the technique permits the measurement of torques lower than $10^{-21} \mathrm{Nm}$ on particles down to picogram mass. (C) 1999 American Institute of Physics.
\end{abstract}

[S0021-8979(99)00508-3]

\section{INTRODUCTION}

Asbestos is a broad term applied to a number of fibrous silicate minerals, including chrysotile (a serpentine), crocidolite, and amosite (both amphiboles). It is now well established that these materials, particularly when they are finely fragmented, pose a serious health hazard. Indeed, the high rates of disease associated with the inhalation of asbestos resulted in a statement by the U.S. National Institute for Occupational Safety and Health that there is no evidence for a threshold or "safe" level of asbestos exposure. ${ }^{1}$ As the health risks associated with these fibers have become more fully understood, severe restrictions on the use of asbestos and rigorous rules concerning its handling have been introduced worldwide. Despite this, there is evidence of a continuing increase in asbestos-related mortality rates, ${ }^{2}$ which may be due to the presence of asbestos in older buildings or equipment on the one hand, and the exposure to asbestos as a result of removal and disposal operations on the other hand. Consequently, the detection of asbestos, particularly in respirable form, is of considerable importance.

The identification of asbestos can be accomplished using polarized light microscopy or scanning electron microscopy in conjunction with energy-dispersive x-ray analysis. However, these methods provide results only after laborious analysis involving skilled human operators, typically hours or days after sample collection. Despite extensive research, there is no in situ, real-time technique allowing unambiguous detection of airborne asbestos fibers.

Magnetic alignment has first been applied to studying asbestos by Timbrell, ${ }^{3,4}$ with further work done by Riis and Chatfield, ${ }^{5}$ Jones and Gale, ${ }^{6}$ and Willey. ${ }^{7,8}$ The nearly unique response of asbestos fibers to magnetic fields has been used for the alignment of fibers deposited in a small quantity of liquid, followed by an examination under a microscope or using light scattering. Some asbestos fiber types have been

${ }^{a)}$ Electronic mail: z.ulanowski@herts.ac.uk found to align with their long axis parallel to, while others perpendicular to the field. In principle, given recent developments in real time airborne fiber analysis using light scattering, ${ }^{9,10}$ magnetic alignment could become the basis for a technique of asbestos detection.

The mechanism by which asbestos fibers are aligned in magnetic fields has remained unclear despite fairly extensive published work. It has variously been suggested that the alignment is due to: the presence of magnetite particles in the chrysotile fibers, "the presence of paramagnetism in the direction of the fiber axis" or normal to the axis in amphibole asbestos $^{4}$ and anisotropy of magnetic susceptibility. ${ }^{5}$ Also, the existence of a permanent magnetic moment has been implied. ${ }^{7,11}$ A further possibility is that the alignment is due to shape anisotropy. Since quantitative data which would allow the prediction of alignment dynamics of asbestos fibers in magnetic fields were also lacking, it was essential to determine both the mechanism by which the fibers were aligned and the strength of the effect.

The microstructure of all types of asbestos is complex but quite different in the two types considered here. Chrysotile, a fibrous silicate from the serpentine group, takes the form of "scrolled" sheets (with scroll axis parallel to the length of the fibers) containing a layer of $\mathrm{SiO}_{4}$ tetrahedra and an octahedral cation (mainly $\mathrm{Mg}$ ) layer. ${ }^{12,13}$ Some of the octahedral sites (typically less than 2\%) are occupied by iron, which can be ferric or ferrous. ${ }^{14}$ A little ferric iron is also found in the tetrahedral $(\mathrm{Si})$ sites. ${ }^{15}$ A significant proportion of iron is also present in the form of magnetite inclusions, ${ }^{13,14,16}$ although data obtained from bulk samples may not be applicable to single fibers. Crocidolite, the fibrous form of riebeckite, has a structure consisting of parallel stacks of long ribbons, each containing an octahedral cation layer sandwiched between two silica double chains, and the unit cell is monoclinic. Some cation sites are occupied by $\mathrm{Fe}^{2+}$ and $\mathrm{Fe}^{3+} \cdot{ }^{12,17}$ The low level of cation substitution by iron in chrysotile ensures that magnetic interactions are weak and that the magnetic susceptibility is low $\left(3 \times 10^{-4}\right.$, SI 
units). ${ }^{16}$ In crocidolite, which has a high iron content (typically about $15 \%),{ }^{16}$ the $\mathrm{Fe}$ ions are more strongly coupled than in chrysotile, but ferromagnetic ordering does not occur until $30 \mathrm{~K}^{17}$ The susceptibility is an order of magnitude higher than in chrysotile $\left(3 \times 10^{-3}\right) .{ }^{12,16}$ The highly asymmetric structures of chrysotile and crocidolite admit the possibility of magnetic anisotropy. While the unit cell of chrysotile is not uniaxial, the overall structure can be expected to lead to uniaxial properties, including axisymmetric susceptibility. It is less certain whether crocidolite can exhibit gross uniaxial symmetry but aggregate fibers can in principle show uniaxial characteristics, as the azimuthal orientations of individual silicate ribbons can vary within thicker fibers.

Due to the existence in asbestos of magnetic impurities, such as magnetite grains, and the possible simultaneous presence of fibers which align at right angles to each other, measurements had to be carried out on single fibers, rather than on bulk samples. The difficulty posed by the need to measure extremely small torques was overcome by the application of a straightforward method which, to the authors' best knowledge, has not been used before. The method relies on counteracting the torques exerted on fibers by a magnetic field and by viscous drag in a rotating fluid.

\section{METHODS}

The torque on a paramagnetic, axisymmetrically anisotropic object without a permanent magnetic moment and placed in a uniform magnetic field of intensity $H$ can be written as

$$
\Gamma_{a}=(1 / 2) \mu_{0} V H^{2} \Delta \chi \sin 2 \theta,
$$

where $\mu_{0}$ is vacuum permeability, $V$ object volume, $\theta$ the angle between the axis of the susceptibility ellipsoid and the direction of the external magnetic field, and $\Delta \chi$ anisotropy of volume susceptibility, defined as the difference between the susceptibilities parallel and perpendicular to the axis of the ellipsoid.

Besides magnetocrystalline anisotropy, shape anisotropy may be present. The magnitude of the latter can be calculated for objects such as prolate ellipsoids. The effective susceptibility of an object characterized by an intrinsic (crystalline) volume susceptibility $\chi$ and for a field applied along direction $i$ is $\chi_{i}=\chi /\left(1+N_{i} \chi\right)$, where $N_{i}$ is the demagnetization factor in the same direction. ${ }^{18}$ For a prolate ellipsoid, the demagnetization factor along the major axis is

$$
N_{1}=\left(\frac{1}{\epsilon^{2}}-1\right)\left(\frac{1}{2 \epsilon} \ln \frac{\epsilon+1}{\epsilon-1}-1\right),
$$

where $\epsilon$ is the eccentricity of the ellipsoid. The factors perpendicular to the major axis are $N_{2}=N_{3}=\left(1-N_{1}\right) / 2 .{ }^{18}$ The shape anisotropy in this case is

$$
\begin{aligned}
\Delta \chi_{s}=\chi_{1}-\chi_{2} & =\frac{\chi}{1+N_{1} \chi}-\frac{\chi}{1+N_{2} \chi} \\
& \approx \frac{\chi^{2}}{2\left(1 /\left(1-3 N_{1}\right)-\chi / 2\right)},
\end{aligned}
$$

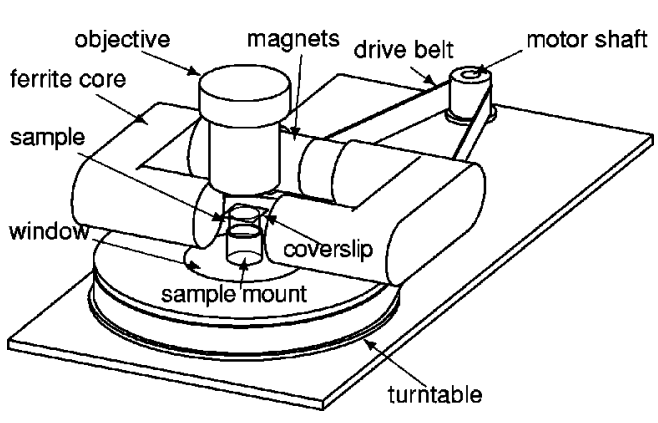

FIG. 1. Rotating stage forming the basis of the torque magnetometer for the measurement of magnetic anisotropy. Only the objective of the optical microscope is shown.

the approximation being valid if $\chi \ll 1$. For slender cylinders, a limiting form of expression (2) can be used by setting $\epsilon$ $=1$, which leads to $N_{1}=0$ and $N_{2}=N_{3}=1 / 2$. The shape anisotropy then becomes

$$
\Delta \chi_{S C}=\chi_{1}-\chi_{2}=\frac{\chi}{1+2 / \chi} \approx \frac{\chi^{2}}{2}
$$

provided again that $\chi \ll 1$.

To measure the torque exerted on single asbestos fibers by magnetic fields a system was constructed which allowed balancing the forces due to magnetism with those due to viscous drag in a rotating, isopycnic liquid (Fig. 1). A small $(70 \mu \mathrm{l})$, transparent, polypropylene container holding a suspension of asbestos fibers was positioned in the center of a rotating stage. The container was located between the poles of a stationary magnet constructed from up to four small, rare-earth, permanent magnets and two U-shaped ferrite cores. The stage could be rotated using a stepper motor. The whole assembly was placed under an optical microscope equipped with a $\times 8$ objective lens, $\times 20$ eyepieces, a graticule and a protractor, to allow the observation of the fibers and the measurement of their sizes and orientation angles.

The container was partially filled with a pure immersion liquid and some suspension of the fibers under study was added to it, in one of two ways. For chrysotile, a discontinuous density gradient was formed in the container. First, the container was half filled with a liquid of density slightly smaller than the density of chrysotile and with some fibers already suspended in it. Then a liquid of higher density was slowly injected under the first layer using a syringe with a blunt hypodermic needle. Both liquids were mixtures of diiodomethane and iodoethane (Fisher Scientific, Loughborough, UK). For crocidolite, because of its density being nearly equal to that of the chosen liquid, a gradient was not used. Instead, a suspension of the fibers in diiodomethane was carefully layered over pure diiodomethane. The container was then covered with a small microscope cover-slip in both cases.

The suspended fibers would undergo alignment as a result of the action of the magnetic field produced by the magnet. Rotation of the stage could then be used to subject the fibers to a torque due to viscous drag. Once an equilibrium was reached (provided that the hydrodynamic torque did not exceed the maximum torque produced by the magnetic field), different magnetic properties of individual fibers could be 
expected to result in different fiber orientation angles, inasmuch as the torque due to the magnetic field was anticipated to be angle dependent. Moreover, the orientation angle would be a function of the rotation rate of the stage, since the torque due to the viscous drag was roughly proportional to that rate. The hydrodynamic torque could be calculated from the rotation rate, the viscosity and density of the immersion liquid, and the dimensions of any given fiber, thus providing a value for the torque due to the magnetic field at the observed orientation angle. The angular dependence of the torque could be determined by varying the rotation rate. The present measurement system can thus be thought of as a torque magnetometer characterized by a constant but adjustable torque rather than a fixed spring constant.

The samples used were of UICC (Union Internationale Contre le Cancer) standard respirable asbestos: crocidolite and Canadian chrysotile. Submilligram amounts of the fibers were dispersed in an appropriate immersion liquid, then filtered through a $70 \mu \mathrm{m}$ nylon mesh. The densities were taken to be $3.335 \times 10^{3} \mathrm{~kg} \mathrm{~m}^{-3}$ for crocidolite and 2.55 $\times 10^{3} \mathrm{~kg} \mathrm{~m}^{-3}$ for chrysotile. The dynamic viscosity and the density of the immersion liquids used were, respectively: $2.8 \times 10^{-3} \mathrm{~kg} \mathrm{~m}^{-1} \mathrm{~s}^{-1}$ and $3.325 \times 10^{3} \mathrm{~kg} \mathrm{~m}^{-3}$ for diiodomethane, $0.59 \times 10^{-3} \mathrm{~kg} \mathrm{~m}^{-1} \mathrm{~s}^{-1}$ and $1.95 \times 10^{3} \mathrm{~kg} \mathrm{~m}^{-3}$ for iodoethane. For suspending chrysotile fibers, the isopycnic solution containing diiodomethane and iodoethane in a $55 / 45$ weight ratio had a calculated viscosity of 1.55 $\times 10^{-3} \mathrm{~kg} \mathrm{~m}^{-1} \mathrm{~s}^{-1}$. The magnetic flux density at the sample was $0.047 \mathrm{~T}$ for crocidolite and $0.20 \mathrm{~T}$ for chrysotile.

The hydrodynamic torque can be derived from the classic expression describing the viscous drag coefficient $c_{D}$ for the case of laminar flow past an infinite circular cylinder: ${ }^{19}$

$$
c_{D}=\frac{8 \pi}{\operatorname{Re}\left(\frac{1}{2}-\gamma+\ln \frac{8}{\operatorname{Re}}\right)} \approx \frac{8 \pi}{\operatorname{Re} \ln \frac{7.4}{\operatorname{Re}}},
$$

where $\operatorname{Re}=\nu d \rho / \mu$ is the Reynolds number, $\nu$ fluid velocity, $d$ cylinder diameter, $\rho$ the density, and $\mu$ the dynamic viscosity of the fluid, and $\gamma$ is Euler's constant. The drag per unit length of the cylinder then becomes ${ }^{19}$

$$
F_{D} \approx \frac{4 \pi \nu \mu}{\ln (7.4 \mu / \rho \nu d)} .
$$

The viscous drag torque on a cylinder rotating about an axis of symmetry perpendicular to the cylinder can be obtained by integrating Eq. (6) over the length of the cylinder:

$$
\begin{aligned}
\Gamma_{D}=2 \int_{0}^{1 / 2} F_{D} r d r & =8 \pi \mu a^{3} \omega \int_{3 \ln (2 a / L)}^{\infty} \frac{e^{-x}}{x} d x \\
& =8 \pi \mu a^{3} \omega E_{1}\left(3 \ln \frac{2 a}{L}\right),
\end{aligned}
$$

where $a \approx 7.4 \mu / \rho \omega d$ and $\omega$ is the angular velocity, $L$ the length of the cylinder, and $E_{1}($.$) the exponential integral.$ Since the latter does not have a convenient closed-form solution, it was approximated on the interval $(25,60)$ using Chebyshev series, giving

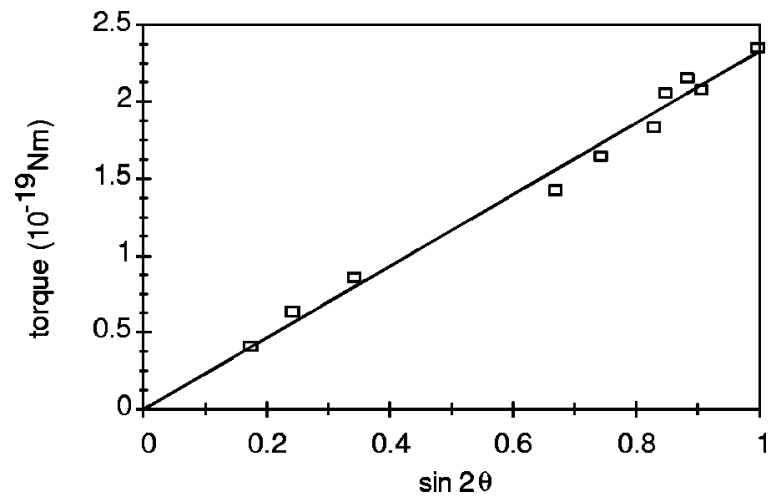

FIG. 2. Measured torque on a single crocidolite fiber as a function of $\sin 2 \theta$, where $\theta$ is the angle between the long axis of the fiber and the direction of the magnetic field. The line is a least squares fit with an intersect of 0. Fiber length was $3 \mu \mathrm{m}$, thickness $0.3 \mu \mathrm{m}$ and flux density $0.047 \mathrm{~T}$.

$$
E_{1}(y)=\exp \left(-2.2271-1.0487 y+0.28909 \times 10^{-3} y^{2}\right),
$$

where $y=3 \ln (2 a / L)$.

For calculations of fiber orientation in air, an equation of motion taking into account the torques due to inertia as well as magnetic anisotropy and viscous drag was solved numerically. However, because of a wide range of angular velocities present (including a singularity at $\omega=0$ ), the expressions (7) and (8) were not used. Instead, the viscous drag was linearized by inserting a constant velocity $v=\omega L / 2$ (velocity at the tip of the rotating cylinder) under the logarithm in Eq. (6) prior to the integration, resulting in the following expression for the aerodynamic torque:

$$
\Gamma_{D} \approx 0.98 \frac{\pi \mu L^{3} \omega}{3 \ln (14.8 \mu / \rho d \omega L)},
$$

where the coefficient 0.98 is introduced to correct for errors due to the linearization.

\section{RESULTS AND DISCUSSION}

Both chrysotile and crocidolite fibers aligned mainly with their long axis along the direction of the field. They could assume either of the two possible parallel orientations without apparent preference. Moreover, the only alignment angles at which fibers could be maintained indefinitely by rotating the turntable were less than $45^{\circ}$ relative to the field. Such behavior cannot be expected if the alignment is due to a permanent magnetic moment but is consistent with the presence of anisotropy of magnetic susceptibility or shape anisotropy. To further test this hypothesis, several torque measurements at different angles were made on individual fibers. Typical results are shown in Fig. 2, where it is clearly seen that the torque is proportional to $\sin 2 \theta$, according to the expectations. Therefore, further measurements and calculations were carried out on the assumption that the observed alignment was due to anisotropy and could be described by the general relationship (1). The distributions of the measured anisotropy of magnetic susceptibility of chrysotile and crocidolite are shown in Fig. 3. The average values of the volume anisotropy were $0.40 \times 10^{-6}$ and $83 \times 10^{-6}$ (SI units) for chrysotile and crocidolite, respectively. 


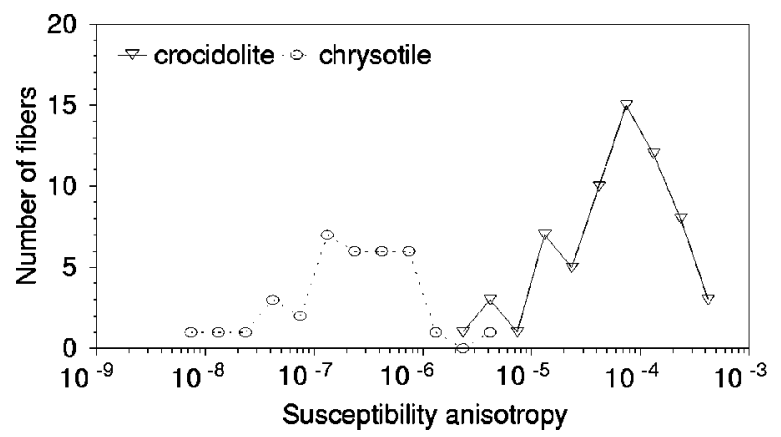

FIG. 3. Distribution of measured anisotropy of volume susceptibility of single asbestos fibers (expressed in nondimensional SI units). The flux density was $0.047 \mathrm{~T}$ for crocidolite and $0.2 \mathrm{~T}$ for chrysotile.

The torque due to shape anisotropy cannot be distinguished experimentally in a straightforward manner from that produced by magnetocrystalline anisotropy, as outlined in the previous section. However, the magnitude of the shape anisotropy, and hence the torque due to it, can be calculated. For this purpose, the fibers were assumed to be slender cylinders. The anisotropy was then calculated using expression (4) taking $3.2 \times 10^{-3}$ and $3.0 \times 10^{-4}$ for the volume susceptibilities of crocidolite ${ }^{11,16}$ and chrysotile, ${ }^{16}$ respectively, and $-1.46 \times 10^{-5}$ and $-1.2 \times 10^{-5}$ for those of the corresponding media (immersion liquids). The calculated shape anisotropy was $5.1 \times 10^{-6}$ for crocidolite and $4.7 \times 10^{-8}$ for chrysotile. For comparison, similar computations were done assuming that the fibers were prolate ellipsoids of equal volume and aspect ratio (i.e., the major and minor semiaxes of the ellipsoids were obtained by multiplying the measured length and thickness, respectively, by $\left.(3 / 16)^{1 / 3}\right)$. The anisotropy was then calculated using expression (3). The average anisotropy values were $5.0 \times 10^{-6}$ and $4.1 \times 10^{-8}$, respectively, for crocidolite and chrysotile-within a few percent of the values calculated assuming cylindrical shape.

The distribution of the ratio of the measured anisotropy to the calculated shape anisotropy is given in Fig. 4. For both chrysotile and crocidolite, the ratio was much greater than one for the majority of the individual fibers studied, i.e., the observed alignment was stronger than that expected from shape anisotropy alone. Hence, it appears that the alignment of the fibers was due to the anisotropy of magnetic suscep-

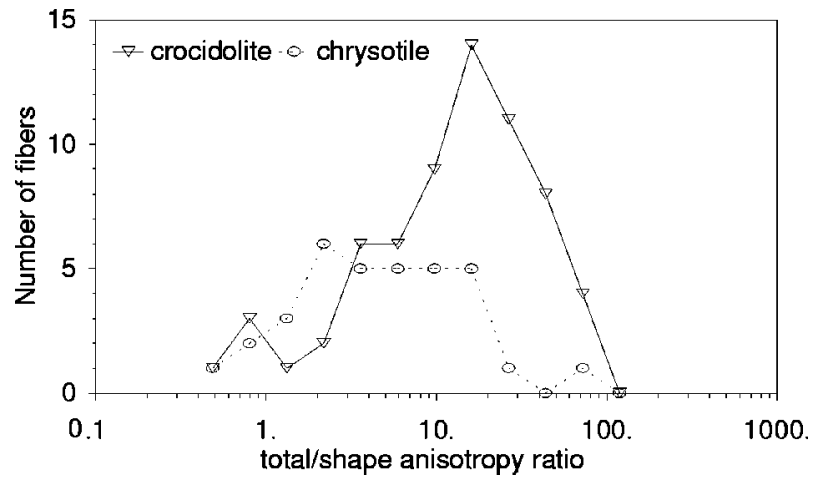

FIG. 4. Distribution of the ratio of the measured anisotropy to the calculated shape anisotropy for single fibers of crocidolite and chrysotile. The fibers were assumed to be slender cylinders. tibility, principally its crystalline component. For crocidolite, the magnitude of the crystalline component of anisotropy was not inconsistent with, if somewhat lower than, the results of low-temperature, high field measurements indicating anisotropy of the order of $10 \%$ for bulk samples of Australian crocidolite, ${ }^{17}$ assuming the magnetic susceptibility of about $3 \times 10^{-3} \cdot 11,12,16$

The width of the observed distributions of anisotropy may be due to a combination of several factors. The measurement of the thickness of the fibers was inherently inaccurate because of limitations of optical microscopy. Since estimates of the hydrodynamic torque based on expression (7) are approximately inversely proportional to the square of the thickness, they may be associated with large errors. Further errors stem from the "slender fiber" assumption underlying the expression for the drag coefficient (5). On the other hand, the measured anisotropy did not extend substantially below the theoretical minimum, as represented by the shape anisotropy (Fig. 4), indicating that the errors were small. The remaining variation might not have been entirely due to the anisotropy of the crystalline matrix of the fibers, since bulk samples of both crocidolite and Canadian chrysotile contain significant amounts of ferrimagnetic impurities, notably grains of magnetite. ${ }^{13,16}$ The flux density used for crocidolite was below that required to achieve full saturation, so magnetite impurities could, in principle, have contributed to the measured anisotropy. However, fine crocidolite fibers, in contrast to bulk samples, are unlikely to contain within them the large pseudosingle-domain magnetite grains which probably dominate the low field magnetic properties of crocidolite. ${ }^{16}$ It is more difficult to draw clear conclusions on the origins of the anisotropy in chrysotile since this material, at least as far as bulk samples are concerned, is likely to contain magnetite grains with a wider distribution of sizes, ${ }^{13,16}$ but the contribution from magnetite was reduced by the higher field used during the measurements.

To establish whether magnetic alignment would be useful as a means of characterization of airborne fibers in flow, reorientation times for asbestos fibers in air were calculated from the measured anisotropy. The equation of motion, taking into account torques due to the anisotropy (1), viscous drag (9), and fiber inertia, as outlined in the preceding section, was integrated numerically. It was assumed that the fibers had to undergo a $10^{\circ}$ reorientation and that their initial angle relative to the field was $45^{\circ}$. The distribution of calculated reorientation times is shown in Fig. 5. Average reorientation times calculated for the measured asbestos fibers were $1.8 \mathrm{~ms}$ for chrysotile and $0.14 \mathrm{~ms}$ for crocidolite at a flux density of $0.5 \mathrm{~T}$. Such short reorientation times should allow the detection of the realignment of airborne fibers in flow by measuring their orientation angles using light scattering ${ }^{9,10}$ and, consequently, make possible the discrimination between asbestos and other fiber types. Chrysotile and crocidolite may also be distinguishable because of small overlap between the predicted distributions for these two materials (Fig. 5). The latter capability is important because 


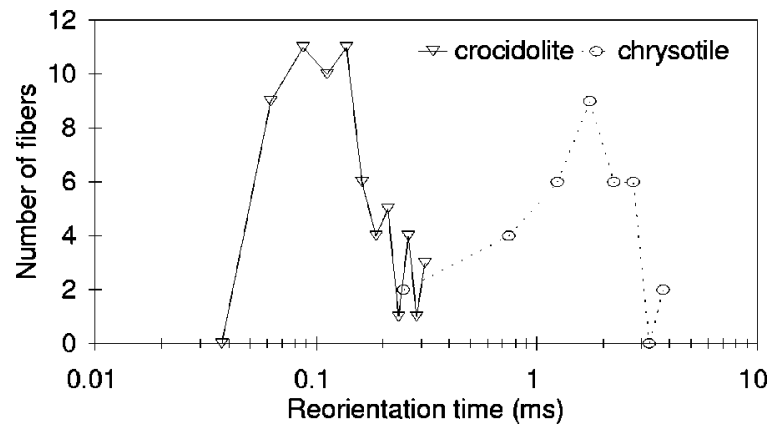

FIG. 5. Distribution of calculated reorientation time for asbestos fibers subjected to a magnetic field in air. The flux density was $0.5 \mathrm{~T}$, the initial angle with respect to the field $45^{\circ}$, the final angle $35^{\circ}$.

there is evidence for an increasing pathogenicity gradient running from chrysotile, through various amphibole forms, to crocidolite. ${ }^{20,21}$ A summary of the properties of the asbestos fibers is given in Table I.

\section{CONCLUSIONS}

The alignment of crocidolite and chrysotile fibers in magnetic fields was found to be mainly due to the crystalline component of the anisotropy of magnetic susceptibility, estimated at $0.35 \times 10^{-6}$ and $78 \times 10^{-6}$ for chrysotile and crocidolite, respectively. Fiber shape effects contributed on average about $6 \%$ and $10 \%$ to the measured anisotropy of crocidolite and chrysotile, respectively. There was no evidence of significant permanent magnetization. Some of the observed anisotropy in chrysotile may have its origin in the presence of small magnetite inclusions; this is less likely to be the case for crocidolite.

Magnetic alignment of asbestos fibers can now be regarded as a strong candidate for use in real-time detection of airborne asbestos fibers. Combining a method of measuring the orientation of airborne fibers in flow ${ }^{9,10}$ with magnetic alignment should allow the discrimination between asbestos and other types of fibers. Amphibole asbestos fibers especially should undergo strong reorientation at flux densities as low as $0.5 \mathrm{~T}$. Detecting chrysotile and distinguishing it from other types of asbestos may also be technically feasible.

TABLE I. Summarized properties of asbestos fibers.

\begin{tabular}{lcc}
\hline \hline \multicolumn{1}{c}{ Property } & Chrysotile & Crocidolite \\
\hline Mean length $(\mu \mathrm{m})$ & 16.7 & 29.7 \\
Mean diameter $(\mu \mathrm{m})$ & 2.5 & 1.7 \\
$\begin{array}{l}\text { Susceptibility anisotropy }\left(\times 10^{-6}\right) \\
\quad \text { measured }\end{array}$ & 0.40 & 83 \\
$\quad$ shape $^{\mathrm{a}}$ & 0.047 & 5.1 \\
$\quad$ magnetocrystalline $^{\mathrm{b}}$ & 0.35 & 78 \\
$\quad \begin{array}{l}\text { Reorientation time in air }(\mathrm{ms})^{\mathrm{c}} \\
\quad \text { mean } \\
\quad \text { maximum }\end{array}$ & 1.8 & 0.14 \\
$\quad$ minimum & 4.0 & 0.59 \\
\hline
\end{tabular}

${ }^{a}$ Calculated for slender cylinders.

${ }^{b}$ Difference between the measured and the shape anisotropy.

${ }^{\mathrm{c}}$ Calculated for each fiber taking as parameters the dimensions and the measured anisotropy of the fiber and assuming flux density $0.5 \mathrm{~T}$, the initial angle with respect to field $45^{\circ}$, the final angle $35^{\circ}$.
Previous studies indicate that very few respirable fiber types can be confused with asbestos on the basis of their magnetic properties. ${ }^{3,8}$ It is indeed highly unlikely that glassy fibers would exhibit magnetic properties similar to those of asbestos unless they contained significant quantities of iron compounds. Crystalline fibers, however, can show anisotropy and could in principle be confused with asbestos. An example is carbon fiber ${ }^{22,23}$ but its occurrence is not likely to be frequent. Also, the anisotropy of man-made carbon fibers is nearly an order of magnitude larger than that of crocidolite, ${ }^{23}$ allowing discrimination between these fiber types.

The experimental technique presented here can be used for measuring the magnetic anisotropy of small particles of well-defined shape - as long as the hydrodynamic torque can be calculated. The sensitivity of the technique would be difficult to attain by other means: torques of $10^{-21} \mathrm{Nm}$ or less are easily measurable. In contrast, the sensitivity of some of the best "conventional" torque magnetometers is of the order of $10^{-13}-10^{-14} \mathrm{Nm} .{ }^{24,25}$ Particles with mass as small as a few picograms can be examined by this technique. In addition, the high sensitivity and wide dynamic range allow both low field and high field measurements to be carried out.

\section{ACKNOWLEDGMENTS}

This research was supported in part by grants from the Engineering and Physical Sciences Research Council and the Leverhulme Trust. The authors are indebted to John Hoskins from the Medical Research Council's Toxicology Unit in Leicester for the asbestos samples, to John Kerr for technical assistance and to Adrian Finch for commenting on the manuscript.

${ }^{1}$ P. A. Baron, in Aerosol Measurement, edited by K. Willeke and P. A. Baron (Van Nostrand Reinhold, New York, 1993), pp. 560-590.

${ }^{2}$ J. Peto, J. T. Hodgson, F. E. Matthews, and J. R. Jones, Lancet 345, 535 (1995).

${ }^{3}$ V. Timbrell, Microscope 20, 365 (1972).

${ }^{4}$ V. Timbrell, Ann. Occup. Hyg. 18, 299 (1975).

${ }^{5}$ P. Riis and E. J. Chatfield, NBS Special Publication 619, Proceedings NBS/EPA Asbestos Standards Workshop, Gaithersburg, 1980 (issued 1982), pp. 108-120.

${ }^{6}$ A. D. Jones and R. W. Gale, Ann. Occup. Hyg. 25, 39 (1982).

${ }^{7}$ R. J. Willey, IEEE Trans. Magn. 18, 1794 (1982).

${ }^{8}$ R. J. Willey, J. Appl. Phys. 61, 3214 (1987).

${ }^{9}$ E. Hirst, P. H. Kaye, and J. A. Hoskins, Ann. Occup. Hyg. 39, 623 (1995).

${ }^{10}$ P. Kaye, E. Hirst, and Z. Wang-Thomas, Appl. Opt. 36, 6149 (1997).

${ }^{11}$ R. Bertrand, Ph.D. thesis, CNAA, UK, 1989.

${ }^{12}$ A. A. Hodgson, in Asbestos, edited by L. Michaels (Wiley, Chichester, 1979), pp. 92-114.

${ }^{13}$ G. Stroink, D. Hutt, D. Lim, and R. A. Dunlap, IEEE Trans. Magn. 21, 2074 (1985).

${ }^{14}$ G. Stroink, C. Blauuw, C. G. White, and W. Leiper, Can. Mineral. 18, 285 (1980).

${ }^{15}$ C. Blauuw, G. Stroink, and W. Leiper, Can. Mineral. 17, 713 (1979).

${ }^{16}$ G. Stroink, R. A. Dunlap, and D. Hutt, Can. Mineral. 19, 519 (1981).

${ }^{17}$ A. Moukarika, J. M. D. Coey, and N. V. Dang, Phys. Chem. Miner. 9, 269 (1983).

${ }^{18}$ W. D. Parkinson, Introduction to Geomagnetism (Scottish Academic Press, Edinburgh, 1983).

${ }^{19}$ H. Lamb, Hydrodynamics, 6th ed. (Cambridge University Press, Cambridge, 1932).

${ }^{20}$ J. C. Mcdonald and A. D. Mcdonald, Eur. Respir. J. 9, 1932 (1996). 
${ }^{21}$ M. Roller, F. Pott, K. Kamino, G. H. Althoff, and B. Bellmann, Exper. Toxicol. Pathol. 48, 3 (1996).

${ }^{22}$ M. J. Matthews, M. S. Dresselhaus, G. Dresselhaus, M. Endo, Y. Nishimura, T. Hiraoka, and N. Tamaki, Appl. Phys. Lett. 69, 430 (1996).
${ }^{23}$ V. Timbrell, J. Appl. Phys. 43, 4839 (1972).

${ }^{24}$ D. Zech, J. Hofer, H. Keller, C. Rossel, P. Bauer, and J. Karpinski, Phys. Rev. B 53, R6026 (1996).

${ }^{25}$ F. B. Humphrey and A. R. Johnston, Rev. Sci. Instrum. 34, 348 (1963). 